\title{
Research on Image Network Retrieval Application Based on Swarm Optimization Algorithm
}

\author{
Qingpeng Nie \\ Rizhao Polytechnic Rizhao 276826, China. \\ nieqingpeng187@163.com
}

\begin{abstract}
The traditional image retrieval adopts a text-based method, which requires a lot of manual annotation of the images in the image library and matches according to the keywords when searching. Already not suitable for the modern image network retrieval, this paper proposes a network model based on the particle swarm optimization algorithm, and then establishes a mathematical model based on swarm intelligence. Using images' low-level visual features to retrieve images is the core of content-based image retrieval. Content-based image retrieval is an approximate matching technique that combines computer vision, image processing, and database and other technical achievements in many fields. The feature extraction can be done automatically by computer, avoiding the subjectivity of traditional manual annotation, and can also improve the accuracy. degree.
\end{abstract}

Key words: Particle swarm algorithm, image, network retrieval, color extraction.

\section{INTRODUCTION}

Research on content-based image retrieval has been developed for nearly 20 years. The traditional image search engines on the Internet, such as Google, Yahoo, and MSN, provide relevant image search functions that rely on the index created by annotations of images to implement search. Features. This traditional search method from text to pictures is not really content-based image retrieval. Although it is simple and easy to understand the content of the text in terms of text interpretation, with the explosive growth of the image database, it is difficult to accurately express the exact meaning of the image with words and the workload is huge; the text label contains more subjective factors for the same image. Different people have different understandings, and then give different annotations; text is an abstract description, it is difficult to cover all the information contained in an image, especially low-level information such as color, shape and texture. At the present stage, the extraction of the low-level features of the image mainly focuses on factors such as color, texture, and shape that have a great influence on vision. These lowlevel features of the image are derived from its visual features, without human intervention and interpretation, and can be automatically extracted.

\section{THE DISADVANTAGES OF TRADITIONAL IMAGE RETRIEVAL}

The defects of these methods are mainly reflected in the fact that when the size of the image database is large, it is not possible to manually label the image; the annotation of the text keyword cannot include the visual information of the image, that is, the semantics of the image cannot be dynamically extracted; The content of the image is rich, and different people will have different understandings. Therefore, the use of text annotation for the same image may have different annotation results, which has a greater impact on the accuracy of the search. Using a single color visual feature, although it can overcome the difference between the text annotation and the content represented by the image, the single visual feature only contains a small amount of image meaning and often does not express the image content clearly. 


\section{IMPROVED IMAGE NETWORK RETRIEVAL THROUGH GROUP OPTIMIZATION ALGORITHM}

Studying content features, similarity metrics, and basic search processes used in content-based image retrieval. For the shortcomings of text annotations and single visual features, designing the image to be less sensitive to translation, scaling, and rotation the eigenvectors are used to represent the images, and an image retrieval method based on particle swarm optimization algorithm is proposed. Finally, the proposed retrieval method is simulated.

\section{Introduction to Particle Swarm Optimization}

Particle swarm optimization (PSO) is a typical swarm intelligence optimization algorithm, which is an evolutionary computing technology. It was proposed by Dr. Eberhart and Dr. Kennedy in 1995. The PSO algorithm is inspired by the regular activities of bird colonies, and then uses a simplified model established by swarm intelligence. PSO algorithm has attracted wide attention from experts and scholars in various fields because of its fast convergence speed, high convergence accuracy, easy implementation, and its superiority in solving practical engineering problems. Similar to the genetic algorithm, the PSO algorithm is an optimization algorithm based on population iteration. However, it does not include the operation of genetic algorithm such as crossover and mutation, but the particle follows the optimal particle in the solution space for searching. Although the research on how to effectively apply the particle swarm optimization algorithm to practical engineering problems has achieved certain results.

\section{Particle Swarm Optimization Algorithm}

In order to analyze and test the performance of the particle swarm optimization algorithm, the following classic test functions will be used in this paper. Sphere function:

The Sphere function is a unimodal function.

In the PSO algorithm, the population consists of randomly generated particles in the search space. The size of the initial population is determined by the actual problem. Each particle has a randomly generated velocity vector to determine the direction of its movement and the steps of the movement. Long, the performance of each particle is determined by the fitness value obtained from the fitness function abstracted from the solution problem.

$$
\mathrm{f}(x)=\sum_{i=1}^{n} x_{i}^{2}
$$

In the D-dimensional search space, in a population of $\mathrm{N}$ particles, at the th iteration, the position of particle $\mathrm{i}$ is expressed as $x_{i}(t)=\left(x_{i 1}(t), x_{i 2}(t) \ldots, x_{i D}(t)\right)$.

Its speed is $\mathrm{v}_{i}(t)=\left(\mathrm{v}_{i}(t), \mathrm{v}_{i 1}(t), \mathrm{v}_{i 2}(t) \ldots, \mathrm{v}_{i D}(t)\right.$ The position with the best fitness value experienced by particle $\mathrm{i}$ is denoted as $\mathrm{p}_{i}(t)=\mathrm{p}_{i 1}(t), \mathrm{p}_{i 2}(t) \ldots, \mathrm{p}_{i D}(t)$

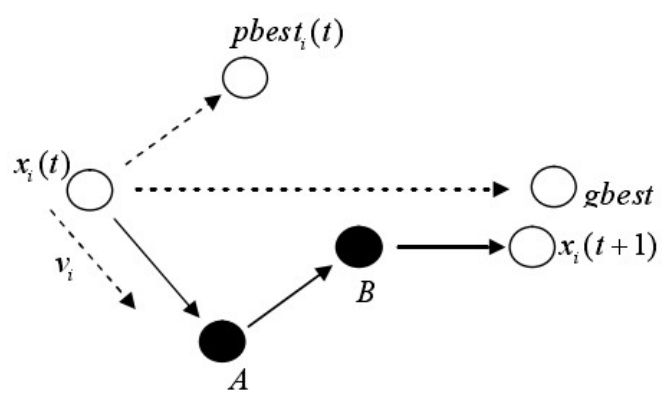

FIGURE 1. Particle Swarm Calculation Method 
It is called the individual optimal solution. The position index with the best fitness value experienced by all particles in the iterative process is denoted by the symbol $\mathrm{g}$, denoted as, $\mathrm{p}_{\mathrm{g}}(t)=\mathrm{p}_{\mathrm{g} 1}(t), \mathrm{p}_{\mathrm{g} 2}(t) \ldots, \mathrm{p}_{\mathrm{g} D}(t)$ Call it the global optimal solution.

Assume that $f(x)$ is the fitness function abstracted from the solution to engineering problems. Taking the minimization problem as an example, the smaller the fitness function value is, the better the fitness of the particles is, that is, the better the performance of the particles.

Its three-dimensional image is shown in Figure 2. It has a global minimum of 0 at the origin of the coordinate.

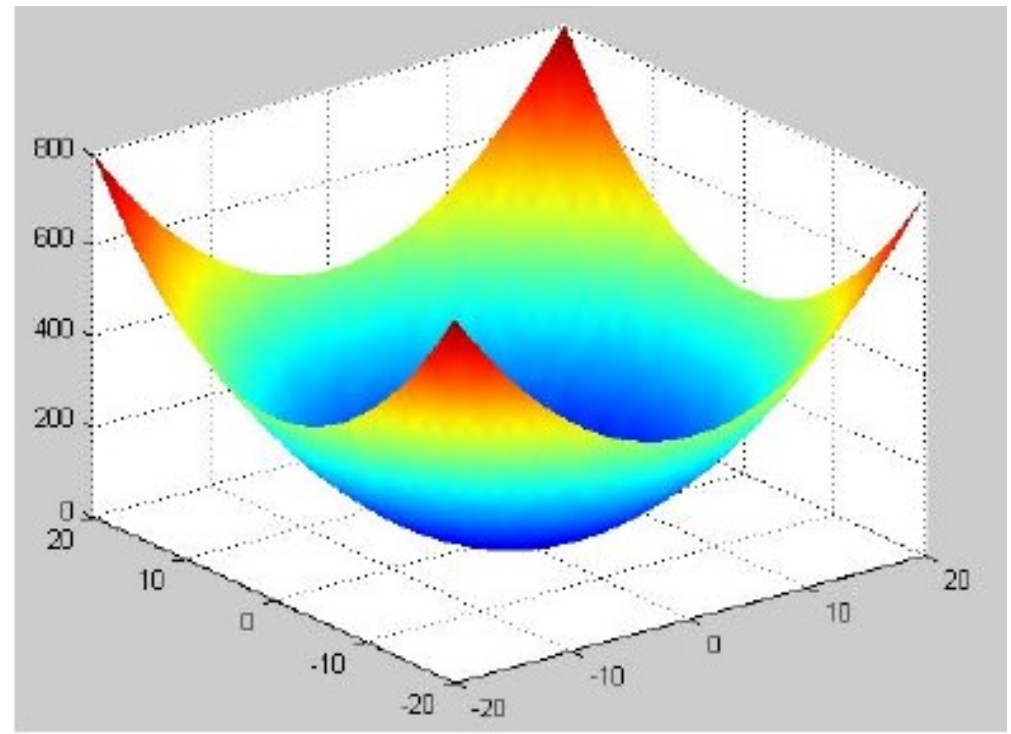

FIGURE 2. Particle swarm optimization algorithm 3D image

The basic particle swarm algorithm initialization process is:

(1) Determine the size of the group based on actual issues.

(2) Initialize the individual optimal solution $\mathrm{p}_{\mathrm{i}}$ and the global optimal solution of the population based on the initial population $\mathrm{p}_{\mathrm{g}}$.Although this method utilizes the characteristics of particles in the process of evolution, the algorithm is optimized and accelerated. The speed of convergence of the algorithm.

\section{CONTENT-BASED IMAGE RETRIEVAL}

Using images' low-level visual features to retrieve images is the core of content-based image retrieval. Contentbased image retrieval is an approximate matching technique that combines computer vision, image processing, and database and other technical achievements in many fields. The feature extraction can be done automatically by computer, which avoids the subjectivity of traditional manual annotation. Generally, it is necessary to provide a target image for the content-based image retrieval process. Subsequently, the system automatically extracts the lowlevel features of the query image, compares it with features in the database, and finally returns an image similar to the target image to the user.

\section{Commonly Used Image Features}

In a broad sense, image features are classified into text features (such as keywords) and visual features (such as shapes, textures, colors, etc.). Among them, visual features can be divided into two categories: general visual features and domain-related visual features. Universal visual features are used to describe common features of images, mainly including information such as color, texture, and shape that are not closely related to the specific 
content of the image; and domain-related visual features are based on the prior knowledge or assumption of the image being described. On the basis of close relevance with practical applications.

Because the color features are well-defined, the extraction method is simple, easy to implement, and has no dependence on the size and orientation of the image, color is one of the most significant features of the image and has been widely used in the earliest image retrieval techniques. In content-based image retrieval techniques, the representation of color features mainly involves the selection of a suitable color space and the selection of appropriate feature extraction methods.

\section{The Color Spaces}

The color space has a variety of representation models, depending on the purpose of the color space can be divided into two categories: visual-oriented color model and hardware-oriented color model. The hardware-oriented color model includes models such as RGB (Red, Green, Blue); the visual-oriented color model includes models such as HSV (Hue, Saturation, Value).

\section{RGB Color Model}

The RGB color model is a universal color standard that obtains various colors by overlapping each other among three color channels: red $(\mathrm{R})$, green $(\mathrm{G})$, and blue $(\mathrm{B})$. It can be expressed as:

$\mathrm{F}=\mathrm{r}[\mathrm{R}]+\mathrm{g}[\mathrm{G}]+\mathrm{b}[\mathrm{B}]$

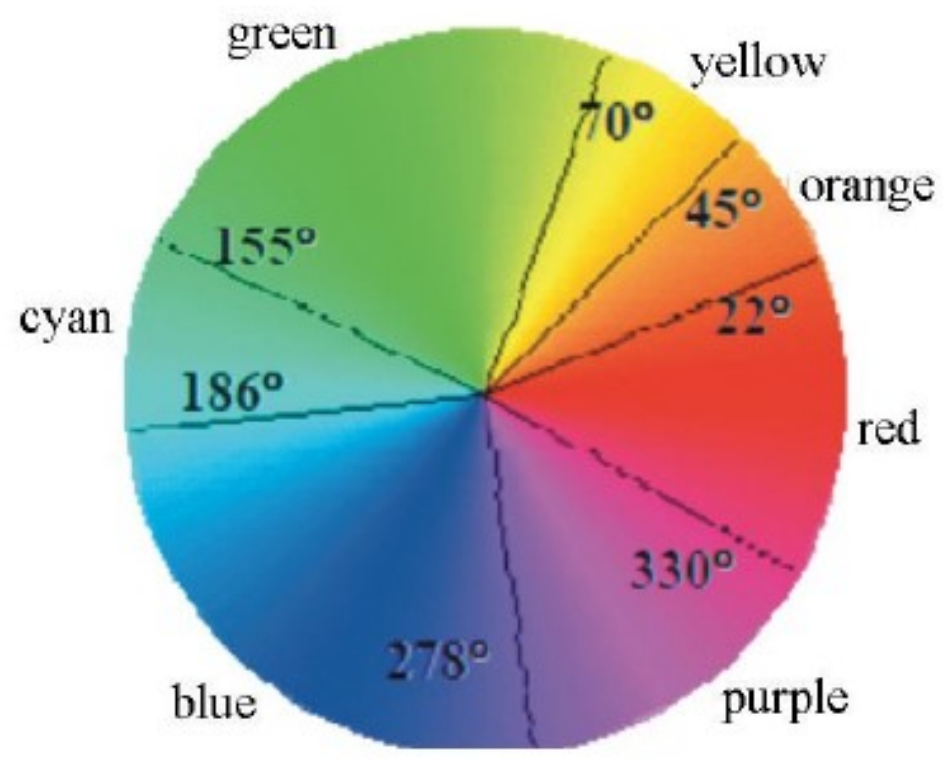

FIGURE 3. Image segmentation

$\mathrm{R}, \mathrm{G}$, and $\mathrm{B}$ respectively represent red, green, and blue color channels. This color model covers most of the colors that can be perceived by vision and is one of the most widely used color models. The RGB color space is a cube in a three-dimensional Cartesian coordinate system The RGB color model is simple and intuitive, and the color image is divided into RGB components and saved. However, the images acquired in the natural environment are easily affected by factors such as natural light and shadows, that is, they are sensitive to brightness. Since the relationship between the RGB color model and brightness is relatively close, that is, the change in brightness will cause the three-color components to change accordingly, this system does not apply to human visual characteristics. 


\section{IMPROVED PARTICLE SWARM OPTIMIZATION IN IMAGE RETRIEVAL}

Image retrieval is a gradual process and should be able to adaptively adjust the direction of retrieval during the retrieval process. The parallel search and learning features of the particle swarm optimization algorithm exactly match the requirements of image retrieval. The parallel search increases the probability of retrieving the target image and improves the retrieval efficiency. The learning characteristics of the particles enable the particles to pass through the target during the retrieval process. By learning information such as images, the search direction is adaptively adjusted and searched in the direction of the target image.

\section{Extraction of Multi-Feature Invariant Moment Vectors}

The moment function is widely used in various image analysis fields such as object classification, pattern recognition, image encoding and reconstruction, and target recognition. The set of moments of the image obtained by the correlation calculation generally represents the global features of the shape in the image and can provide different types of feature information related to the image, such as position, orientation, size, and shape, and another geometric feature information. Image moments are widely used in various areas of image processing, computer vision, and robot technology for target recognition and position estimation with their unique description capabilities. The invariant moment belongs to the statistical characteristics of the image. It has the characteristics of invariance of translation, scaling, and rotation and has been widely used in image recognition.

\section{Geometrical Division of Images}

In image retrieval, segmenting an image not only describes the image as a whole, but also facilitates the description of image features and the discrimination of similarities by appropriately segmenting the image into regions rich in meaning.

In this paper, the image is partitioned into 33-sized image blocks using a block-by-block method.

After image segmentation, image blocks with the same number are divided into the same area. The length and width of image block 1 are respectively one-third of the length and width of the entire image. Images segmented in this way have rotation invariance. Because when observing an image, the information in the four corners of the image is the most likely to be ignored, the image block numbered 1 is recorded as the same region; the information in the middle portion of the image is the first concern, which provides the most important information Therefore, it is marked separately as a region, and when the image is segmented by this method, the image center block occupies a larger area, which is more in line with the general laws of people when they pay attention to the image; the amount of information provided by the image area numbered 2 is introduced. Between image areas 1 and 3 . In the calculation of similarity, the similarity of the image is calculated by assigning different weights to each region in consideration of the importance of information contained in different regions and the contribution to vision.

\section{Calculation of Color Moments}

Higher-order moments are very sensitive to errors in the imaging process, minor deformations, and other factors. Studies have shown that only the description of two-dimensional objects based on moment invariants of second moments is truly independent of image rotation, translation, and scale. In this paper, when calculating the color moment, the second-order color center moment is used, that is, the second-order color moment is calculated for each color channel of each area.

\section{Tonal Quantization}

The calculation of color moments Higher order moments is very sensitive to errors in the imaging process, minor deformations, and other factors. Studies have shown that only the description of two-dimensional objects based on moment invariants of second moments is truly independent of image rotation, translation, and scale. In this paper, when calculating the color moment, the second-order color center moment is used, that is, the second-order color moment is calculated for each color channel of each area. 


\section{Edge Point Calculation}

Shape is one of the most significant visual features of an object and one of the important features of an image. The difficulty of shape extraction technology in image is mainly reflected in the segmentation of the interested part in the figure. The most widely used method is to extract the shape features of an image by finding geometric invariants. Two types of shape description methods are mainly used in image retrieval, namely shape description based on edge detection and shape description method based on regions. This article uses a shape description method based on image edge information.

However, currently using the PSO algorithm for image retrieval methods requires the user to provide relevant feedback, that is, the user needs to select the image satisfying the retrieval requirement according to the convergence result after the algorithm has iterated a certain number of times, and then the algorithm is performed according to the user's feedback result. Follow-up iteration optimization. Although this method ensures the correctness of the retrieval results, the introduction of the feedback mechanism aggravates the user's burden, and the feedback process consumes more time and drastically reduces the retrieval efficiency. This algorithm can be obtained without user feedback. The search results, therefore, further prove that the algorithm can effectively improve the retrieval efficiency.

\section{CONCLUSION}

The defects of traditional text-based retrieval methods and single image feature retrieval methods are analyzed. The commonly used image features and similarity metrics in content-based image retrieval methods are studied. The general flow of content-based image retrieval is described. Aiming at the defects existing in the existing retrieval methods, the image vectors that have less translation, scaling, and rotation sensitivity were designed to represent the images. An improved image retrieval method based on the particle swarm optimization algorithm was proposed. That is, the target image was defined as the global optimal solution of the particle swarm algorithm, the retrieval process is seen as the use of particles in the search space for learning and evolution, and then iteratively search for this optimal solution. The experimental results show that the improved method of image retrieval using the particle swarm optimization algorithm can obtain the final retrieval result quickly, accurately and stably according to the target image.

\section{REFERENCES}

1. Holland J H., et al. Adaptation in natural and artificial systems. Ann Arbor: University of Michigan press, 1975.

2. Dorigo M, Maniezzo V, Colorni A., et al. Ant system: optimization by a colony of cooperating agents [J]. IEEE Transactions on Systems, Man and Cybernetics-Part B, 1996, Vol. 26No. 1p. 29-41.

3. Kirkpatrick S, Gelatt Jr C D, Vecchi M P., et al. Optimization by simulated annealing. Science Vol., 1983, 220p. 671-680.

4. Kennedy J, Eberhart R C., et al. Particle swarm optimization. Proceedings of IEEE International Conference on Neural Networks. Piscatawy, NJ, 1995p. 1942-1948.

5. Shi, Y., Eberhart R.C., et al. A modified particle swarm optimizer. Proceedings of the IEEE International Conference on Evolutionary Computation. Piscataway, NJ, 1998p. 69-73.

6. Shi, Y., Eberhart R.C., et al. Empirical study of particle swarm optimization[C]. Proceedings of the IEEE Congress on Evolutionary Computation. Piscataway, NJ, 1999p. 1945-1950.

7. Yuhui Shi, Eberhart, R.C, et al. Fuzzy adaptive particle swarm optimization. Proceedings of the IEEE Congress on Evolutionary Computation. San Francisco, USA: IEEE, 2011p. 101-106.

8. Eberhart R.C., Shi Y., et al. Tracking and optimal dynamic systems with particle swarms. Proceedings of the IEEE Congress on Evolutionary Computation. San Francisco, USA: IEEE, 2001.p. 94-100.

9. MS Kankanhalli, et al. Cluster-based Color Matching for Image Retrieva. Pattern Recognition, 1996 , Vol. 29(4), p. 701-709.

10. P. J. Angelin., et al. Evolutionary Optimization versus Particle Swarm Optimization: Philosophy and Performance Difference. Annual Conference Center on Evolutionary Programming, San, 1998.p. 243-249. 